\title{
Innovationen brauchen Orte, an denen sie erdacht und erprobt werden
}

$\mathrm{M}$ it der im Herbst 2010 vom IT-Planungsrat beschlossenen Nationalen E-Government Strategie (NEGS) haben sich Bund, Länder und Kommunen zum ersten Mal auf eine gemeinsame Handlungsagenda verständigt. Aus der Perspektive des Jahres 2015 müssen wir uns ehrlicherweise jedoch eingestehen, dass viele der in der NEGS fixierten Ziele bisher nicht erreicht wurden.

\section{$\mathrm{D}$} ie Ursache für den „analogen Tiefschlaf“ - wie eine große deutsche Tageszeitung erst kürzlich den digitalen Entwicklungsrückstand Deutschlands im Bereich des E-Government bezeichnete - ist längst identifiziert: Deutschland hat kein Strategiedefizit, sondern ein Umsetzungsproblem. Die Gründe hierfür sind ebenfalls schon lange bekannt: Denken und Handeln in tradierten Zuständigkeiten, unzureichend entwickelte Strukturen der verwaltungsübergreifenden Zusammenarbeit sowie zeitlich begrenzte politische Handlungslogiken. Im Ergebnis verliert Deutschland viel Zeit und auch Geld. Dementsprechend mahnt Johannes Ludewig, Vorsitzender des Nationalen Normenkontrollrats, immer wieder zu Recht an: „Wir müssen uns über die Ministeriums- und Behördengrenzen hinweg besser organisieren. Nur so können wir die erheblichen Einsparmöglichkeiten und Effizienzgewinne von E-Government wirklich nutzen.“ (Die Welt, 15.3.2015)

$\mathrm{D}$ ie Erkenntnis, dass innovative Vorgehensweisen einen Raum benötigen, in dem sie entwickelt und erprobt werden können, hat im Dezember 2010 die Länder Baden-Württemberg, Hessen und Rheinland-Pfalz sowie den Bund dazu bewogen, gemeinsam mit der Metropolregion Rhein-Neckar (MRN) das Modellvorhaben „Kooperatives E-Government in föderalen Strukturen“ zu initiieren.

\section{I}

Innerhalb der letzten fünf Jahre konnten im Rahmen des Modellvorhabens insgesamt 15 E-Government-Projekte gestartet und teilweise bereits erfolgreich abgeschlossen werden. $\mathrm{Zu}$ den Erfolgen zählen u.a. die gleichzeitige Freischaltung der einheitlichen Behördenrufnummer 115 für 1,7 Millionen Einwohner und der Aufbau eines länderübergreifenden Servicecenters, die Realisierung einer E-Vergabe-Lösung mit über 50 beteiligten Verwaltungen sowie die Erstellung einer Digitalen Straßenkarte als erster Schritt einer regionalen Zusammenarbeit für eine effizientere Verwaltungspraxis in Bezug auf Großraum- und Schwertransporte. Doch noch wichtiger als die konkreten Projekterfolge ist vielleicht die Tatsache, dass mit dem Modellvorhaben ein institutioneller Rahmen geschaffen worden ist, in dem sich eine neue Kultur der Zusammenarbeit entwickeln konnte. Heute ist (fast) allen Beteiligten in der Modellregion Rhein-Neckar klar, dass der Aufbau ei- ner vernetzten Verwaltung nur gemeinsam bewältigt werden kann.

Auch wenn die bisherige Bilanz des Modellvorhabens in der MRN sehr erfreulich ist (und sich im Ergebnis bereits zwei weitere Erprobungsräume in Deutschland konstituiert haben), so sind diese Entwicklungen mit Blick auf die noch vor uns liegenden Herausforde-

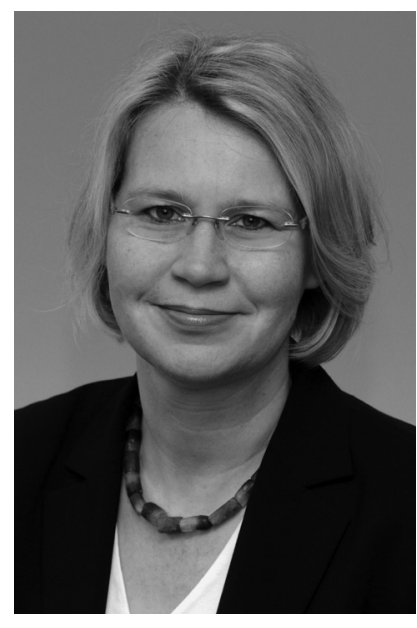
rungen jedoch kaum mehr als ein erster Anfang. Die zunehmende Digitalisierung in allen Lebens- und Arbeitsbereichen sowie die damit verbundenen bedeutenden infrastrukturellen Veränderungen in den Bereichen Energie, Gesundheit, Bildung und Verkehr wird den Modernisierungsdruck für die öffentliche Verwaltung in den nächsten Jahren noch einmal deutlich erhöhen.

$\mathrm{D}$ ie Digitale Agenda der Bundesregierung zeigt einen anspruchsvollen Handlungsrahmen auf, um die vielfältigen Herausforderungen zu bewältigen. Dieser muss jedoch konkretisiert und umgesetzt werden. Dabei reicht es keinesfalls aus, die bisherigen Aktivitäten der verschiedenen Ministerien einfach in das neue Handlungsraster einzuordnen. Was wir brauchen, ist ein neuer Schub der engagierten sachorientierten $\mathrm{Zu}$ sammenarbeit über Verwaltungsebenen und Ressortgrenzen hinweg.

E rprobungsräume und Modellregionen können hierbei einen wichtigen Beitrag leisten. Eine Einschätzung die inzwischen auch der IT-Planungsrat teilt. Zukünftig sollen entsprechende institutionelle Strukturen „zur Erprobung der Praxistauglichkeit bei zukünftigen Gemeinschaftsprojekten und insbesondere bei seinen Koordinierungs- und Steuerungsprojekten“ stärker Berücksichtigung finden (Entscheidung 2014/27). Auf dieser Basis werden sich die vielfältigen Potenziale der neuen digitalen Technologien für den Standort Deutschland sicherlich noch sehr viel besser erschließen lassen, als in den vergangenen fünf Jahren.

Ihre

\section{Christine Brockmann}

Leitung Verwaltungsvereinfachung/E-Government der Metropolregion Rhein-Neckar GmbH 